\title{
An improved EMD method and its application in the characteristic of the gear crack fault analysis
}

\author{
Yunbo ZHU ${ }^{1, a}$, Benli WANG ${ }^{1}$, Na FANG ${ }^{1}$, Jun LI \\ ${ }^{1}$ Wuhan Mechanical Technology College, Wuhan, 430075,China \\ a'E-mail: 573150479@qq.com
}

\begin{abstract}
Keywords: Empirical Mode Decomposition; End Effect; Wave Matching; Crack Fault Simulation; Characteristic Components
\end{abstract}

\begin{abstract}
For the end effect produced in the decomposition process of empirical mode decomposition (EMD), based on the analysis on the subsistent method of the wave matching, a new improved method of empirical mode decomposition is founded using a new cut off condition during the process of the decomposition. Through the analysis on the signal produced by the experiment with the crack fault simulation, the results indicate that the method keeps the change trend of the signal at the endpoint preferably, and the characteristic components of the gear have been decomposed effectively.
\end{abstract}

\section{Introduction}

As a direct aim at data, adaptive and needless to foreordained decomposition basis method which can analyze nonlinear non-stationary signal, after be created by Norden E. Huang in 1998, empirical mode decomposition (EMD) has been widely used in machine system fault diagnosis. But the problem of boundary distortion is the most difficult point in empirical mode decomposition. Especially for complicate signal which contains multi-components, EMD will magnify the fringe effect and seriously submerge the end feature of signal because it needs repeatedly interpolation.

This article used a new improved method to empirical mode decomposition based on wave matching, combining the analysis of amplitude spectrum, after the analysis on the simulation signal, proved the rationality of the method. Moreover, we used the signal from gearbox fault test-bed as the simulation model, through the analysis of amplitude spectrum on IMF components, verified the rationality of the method in this article when it used in practice signal processing.

\section{The calculate of wave prolongation degree}

The foundation of the restraining end effect method based on the thought of wave matching is the signal wave match tracing. Its basal thought is find the most similar wave in the signal to match the wave at the end part and prolong the data, then some local extreme point outside the sampling interval can be gained. The end effect will be released to prolongation area when using EMD decomposes the new data. Afterward cutting off the prolongation part, we can get the exact decomposition from original signal.

The calculation of wave matching degree includes the following processes:

1. Suppose the left endpoint of signal $s(t)$ is $k 0$, let $k 0$ be the origination, choose a curve $s 1(t)$ rightwards, make sure the curve contains at least one local maximum point, one local minimum point and one zero-crossing point, the length is 10 ;

2. In the signal $s(t)$, choose a curve $s 2(t)$ rightwards from the right endpoint of curve $s 1(t)$, let curve s2(t) has the same length with curve s1(t). Suppose P1(t1,u1) and P2(t2,u2) are two points in curve s1(t) and s2(t) separately. Translate s1(t), make the point P1 and P2 curve s2(t) coincide. This is a process for two waves align in the coordinate axis according to a reference point, as shown in Fig.1; 

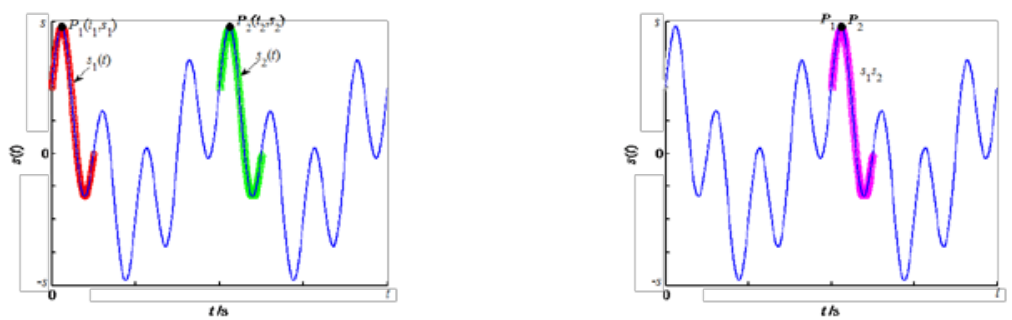

Fig.l Schematic diagram of the waveform matching process

3. After the alignment, namely the new wave is $\mathrm{s}^{\prime} 1(\mathrm{t})$ after Translate $\mathrm{s} 1(\mathrm{t})$. According to the following formula, we can calculate the match degree of s1(t) and s2(t) aim at reference point P1 (or P2):

$$
m\left(x, y, P_{1}\right)=\sum_{i=1}^{N}\left[s_{2}(i)-s_{1}{ }^{\prime}(i)\right]^{2}, \mathrm{~N} \text { is the length of sequence }
$$

\section{The calculation of improved adaptive wave prolongation}

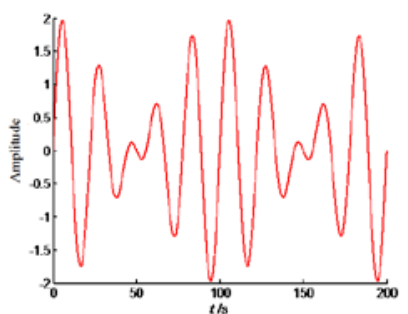

(1.a)

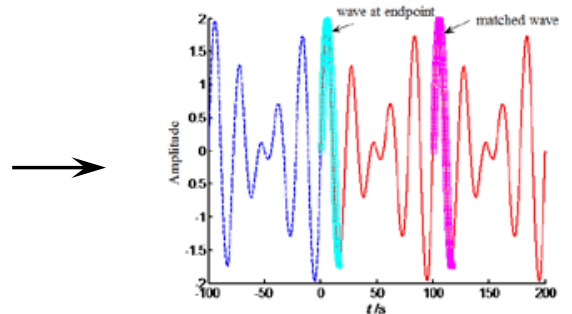

(1.b)

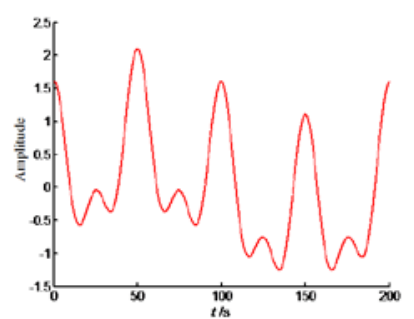

(2.a)

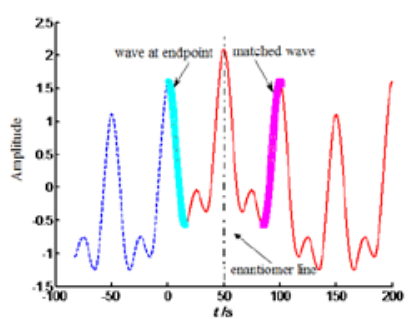

(2.b)

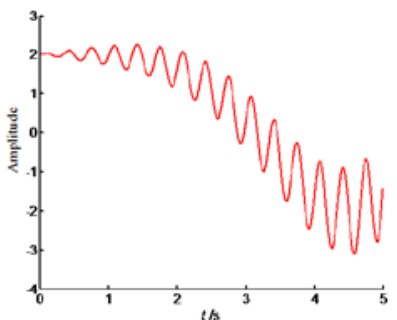

(3.a)

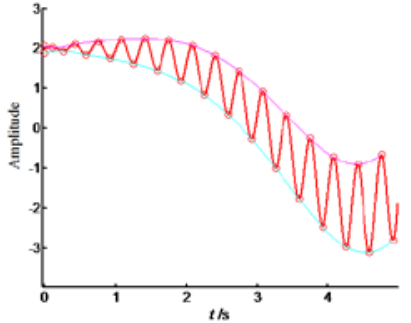

(3.b)

Fig. 2 Analysis on waveform characteristics and the correspond ing extension method

The prolongation wave must fit the change tendency of original signal in the endpoint. So the definition of the changing tendency is the key of prolongation. According to analyze the feature of signal, we know that no matter the signal is periodic or not, if we choose a wavelet in the signal which is similar to the endpoint to prolongate the fringe of the wave, the change tendency of original signal can be protect mostly, so the end effect can be restricted. Even if the wavelet similar to the endpoint can not be found, through calculating the local data in the fringe, we are able to gain the ideal processing result. Based on the above theory and the method to calculate the match degree, according to analyze the feature of signal, the relevant method to wave prolongation (take left endpoint prolongation for example) respectively are:

1. If the wave of signal is periodic, and the characteristic of symmetry is distinctness, as shown in Fig. 2(1.a), in this kind of signal, the match wave for endpoint can be found easily, the signal wave between match wave and endpoint wave is shown in Fig. 2(1.b). After wave translation, the matched wave in the endpoint can be gained, just as the dashed line in Fig. 2(1.b).

2. When the symmetry characteristic of the wave is not distinctness or the wave has not symmetry characteristic, but it has dissymmetry characteristic, the wave can be considered as periodic signal or a part of periodic signal, as shown in Fig. 2(2.a). We can see from the figure, in the interior of the signal wave, it is hard to find the ideal matching wave for the endpoint, but it is easy to find the enantiomorphous matching wave, as shown in Fig. 2(2.b). The matching wave in the right endpoint can be considered as the prolongation wave for the endpoint, just as the dashed line in Fig. 2(2.b). 
3. If the signal wave is non-periodic, but the wave that can match the endpoint wave can be found in the interior of the signal, we can define the prolongation wave through the above two methods.

4. If the signal wave is non-periodic, and the wave that can match the endpoint wave can not be found in the interior of the signal, as shown in Fig. 2(3.a). We can process the signal endpoint by appointing the maximum point and the minimum point. The following methods often are used: regard the value of the endpoint as the maximum point or the minimum point directly; regard the maximum and the minimum nearby the endpoint of the signal as the maximum and the minimum of the endpoint; regard the mean value of the two contiguous maximums in the leftmost point of signal as the maximum of the left endpoint, regard the mean value of the two contiguous minimums in the leftmost point of signal as the minimum of the left endpoint etc. During the practical application, in order to avoid the case that the envelope annexes the wave curve, it is necessary to combine various methods. Meanwhile, we should consider the calculation efficiency of the signal decomposition.

\section{Measured vibration signal analyze}

In order to prove the rationality of the endpoint prolongation wave method, we design the gear crack fault vibration testing on the simulated gear fault test bench.The gear box for experiment is single-stage transformation, the small gear is the drive gear, the big gear is the driven gear and it is the fault gear, the transformation ratio is $75 / 55$. The experiment gain the vibration signal through acceleration sensor, sampling frequency is $5 \mathrm{k} \mathrm{HZ}$; the sampling size is 20480 points. We use the first 5120 sampling points of the signal for researching.

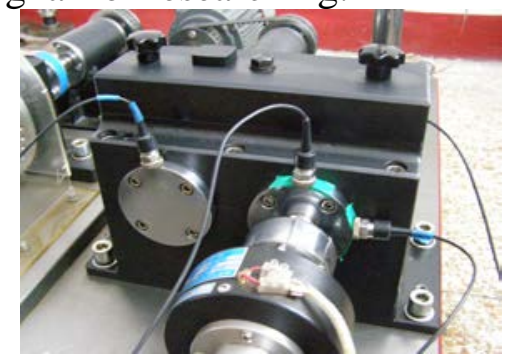

Fig. 3 Gearbox for experiment

The rotating frequency: $f$, meshing frequency: $f_{\mathrm{m}}$ of the experiment gear can be calculated :

$$
f=\frac{n}{60}=\frac{600}{60}=10 \mathrm{~Hz} \quad \text { (2) } \quad f_{m}=\frac{n}{60} \mathrm{Z}=\frac{600}{60} \times 55=550 \mathrm{~Hz}
$$

Analyze the gear crack fault signal through the improved method of endpoint processing, the empirical mode decomposition result is shown in Fig.4(a).we can see from the graphic, 9 IMFs appeared after empirical mode decomposition. If we analyze the fault singal through Rilling EMD method, the empirical mode decomposition result is shown in Fig.4(b), and 12 IMFs appeared after empirical mode decomposition, the remainder IMF is nearly zero.

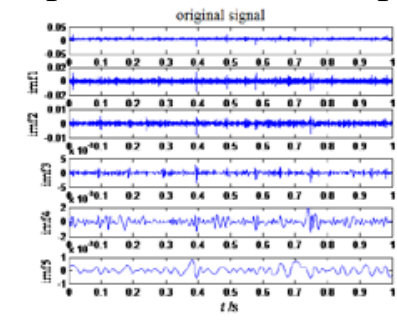

(a) using the improved EMD method

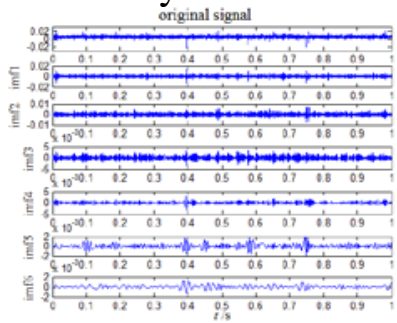

(b) using the Rilling EMD method

Fig. 4 Analysis on the decomposition result of the simulation fault signal

Analyze the IMFs getting from the improved EMD method, the amplitude of spectral peak frequency of the sixth IMF components(as shown in Fig. 4(a)) is $10 \mathrm{~Hz}$, that is corresponding to the rotation frequency of the system. And the peak frequency of the second IMF(as shown in Fig. 5(a)) is $550 \mathrm{~Hz}$ and $600 \mathrm{~Hz}$, the $550 \mathrm{~Hz}$ is corresponding to the meshing frequency of the system,and the $600 \mathrm{~Hz}$ corresponding to the "ghostline" frequency caused by the machining error. 


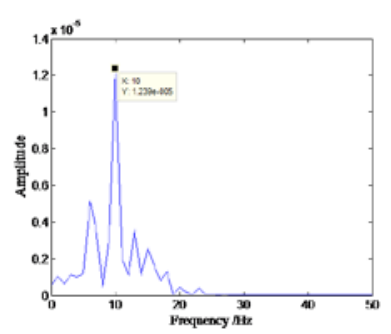

the sixth IMF

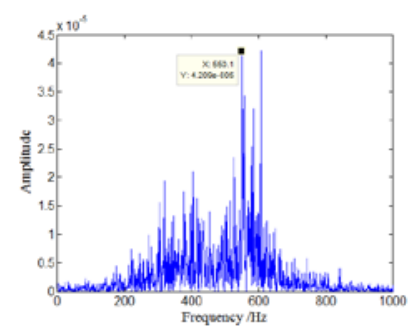

the second IMF

(a) using the improved EMD method

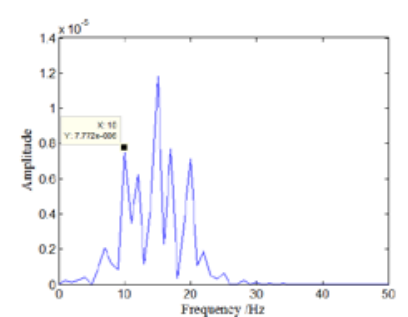

the eighth IMF

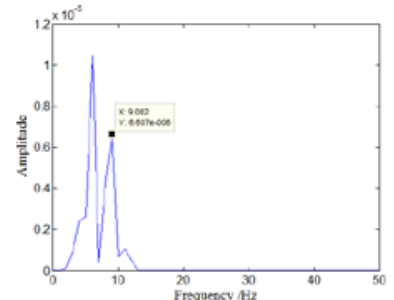

the ninth IMF

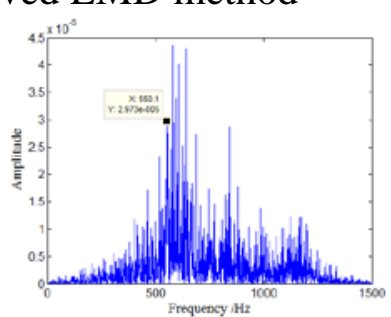

the second IMF

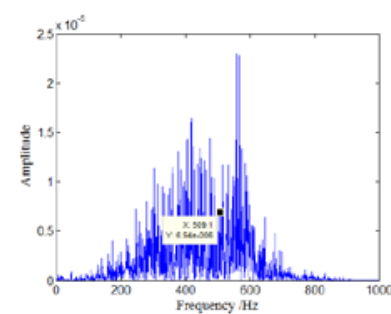

the thrid IMF

(b) using the Rilling EMD method

Fig. 5 Spectral analysis on the decomposition results

Analyze the IMFs(as shown in Fig. 4(b)) getting from the Rilling EMD method, we can find the peak frequency at $10 \mathrm{~Hz}$ in the amplitude of the eighth IMF components(as shown in Fig. 5(b)), but this peak is not the maximum peak in the frequency range of $10 \sim 20 \mathrm{~Hz}$, so we can not get the rotation frequency feature. We can find the peak frequency at $550 \mathrm{~Hz}$ in the second IMF, but this peak is much lower than the "ghostline" frequency, so the feature frequency is hard to recognize. In the amplitude of the third IMF, the peak frequency at $550 \mathrm{~Hz}$ is inundated by other peaks.

\section{Conclusion}

This article found a new improved method of empirical mode decomposition.Compare with the Rilling EMD method ,the proposed method can decompose the characteristic components of the gear system, and it has the high precision and computational efficiency.

\section{References}

[1] N. E. Huang et al. The empirical mode decomposition and the Hilbert spectrum for non-linear and non stationary time series analysis. Proc. Royal Soc. London A, 1998, 454:903-995.

[2] G. Rilling, P. Flandrin, P. Gonçalves, et al. On Empirical Mode Decomposition and its algorithms, IEEE-EURASIP Workshop on Nonlinear Signal and Image Processing NSIP-03, Grado (I), 2003.

[3] N. E. Huang et al. A confidence limit for the Empirical Mode Decomposition and Hilbert spectral analysis. Proc. Royal Soc. London A, 2003, 459:2317-2345.

[4] Zhao Jingping, Huang Daji. Mirror extending and circular spline function for empirical mode decomposition method. Journal of Zhejiang University(Science), 2001, 2(3): 247-252.

[5] Patrick Flandrin, Gabriel Rilling, Paulo Goncalvés. Empirical Mode Decomposition as a Filter Bank [J]. IEEE SIGNAL PROCESSING LETTERS, VOL.11, NO.2, February 2004, 112-114.

[6] Huang $\mathrm{NE}, \mathrm{Wu} \mathrm{M}-\mathrm{L}, \mathrm{Qu} \mathrm{W}$, Long SR. Applications of Hilbert-Huang transform to non-stationary financial time series analysis [J]. Applied Stochastic Models in Business and Industry, 2003, 361(19):245-268.

[7] Guo Mingwei, Ni shihong, Zhu jiahai. HHT/EMD end extension method in vibration signal analysis[J]. Journal of vibration and shock, 2012, 31(8):62-66. 